\title{
On the correlation between convective plume updrafts and downdrafts, lidar reflectivity and depolarization ratio
}

\author{
Fabien Gibert · Juan Cuesta • Jun-Ichi Yano • \\ Nicolas Arnault • Pierre H. Flamant
}

Published online: 6 September 2007

(C) Springer Science+Business Media B.V. 2007

Erratum to: Boundary-Layer Meteorol (2007)

DOI 10.1007/s10546-007-9205-6

There are errors in the placement of some figure captions. In paragraph 6.3, Fig. 8 should display the caption placed with Fig. 10, Fig. 9 should display the caption placed with Fig. 8. Also, in the beginning of paragraph 7, Fig. 10 should show the caption at Fig. 9. Figs. 8-10 with their correct captions beneath are shown below.

The online version of the original article can be found under doi: 10.1007/s10546-007-9205-6

F. Gibert $(\bowtie) \cdot$ J. Cuesta · N. Arnault · P. H. Flamant

Institut Pierre Simon Laplace, Laboratoire de Météorologie Dynamique, Ecole Polytechnique, Palaiseau Cedex 91128, France

e-mail: fabien.gibert@1md.polytechnique.fr

J.-I. Yano

Centre National de Recherche en Météorologie, Toulouse, France 


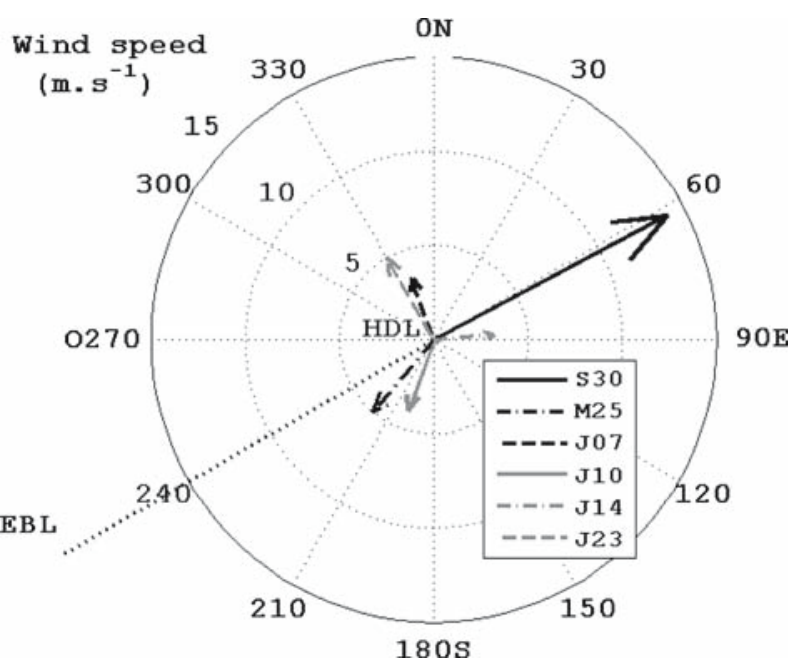

Fig. 8 The CBL mean wind direction and the intensity calculated from the Trappes radiosounding at 1130 UT for the cases defined by various curves. Relative positions of the heterodyne Doppler lidar (HDL) and elastic backscatter lidar (EBL) are also indicated with the two points connected by a thick short-dashed line 

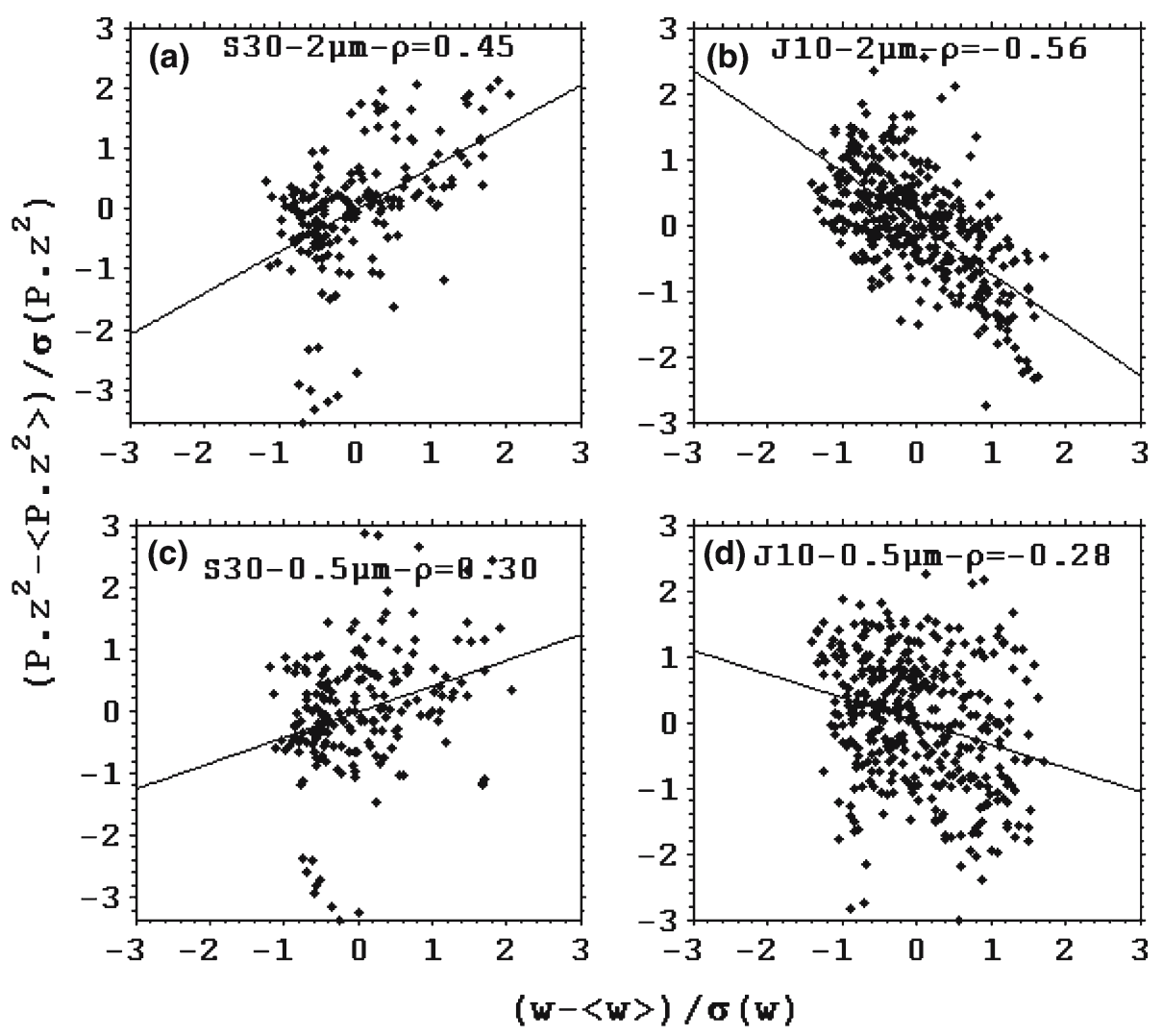

Fig. 9 The same as Fig. 7 but with the CBL height replaced by the normalized lidar reflectivity $\left(P z^{2}-\left\langle P z^{2}\right\rangle\right) / \sigma\left(P z^{2}\right)$ (both from the 2,064 and $532 \mathrm{~nm}$ lidars) against the normalized vertical velocity fluctuations $(w-\langle w\rangle) / \sigma(w)$ at $z_{i} / 2 . \rho\left(w, P z^{2}\right)$ is the calculated correlation coefficient 

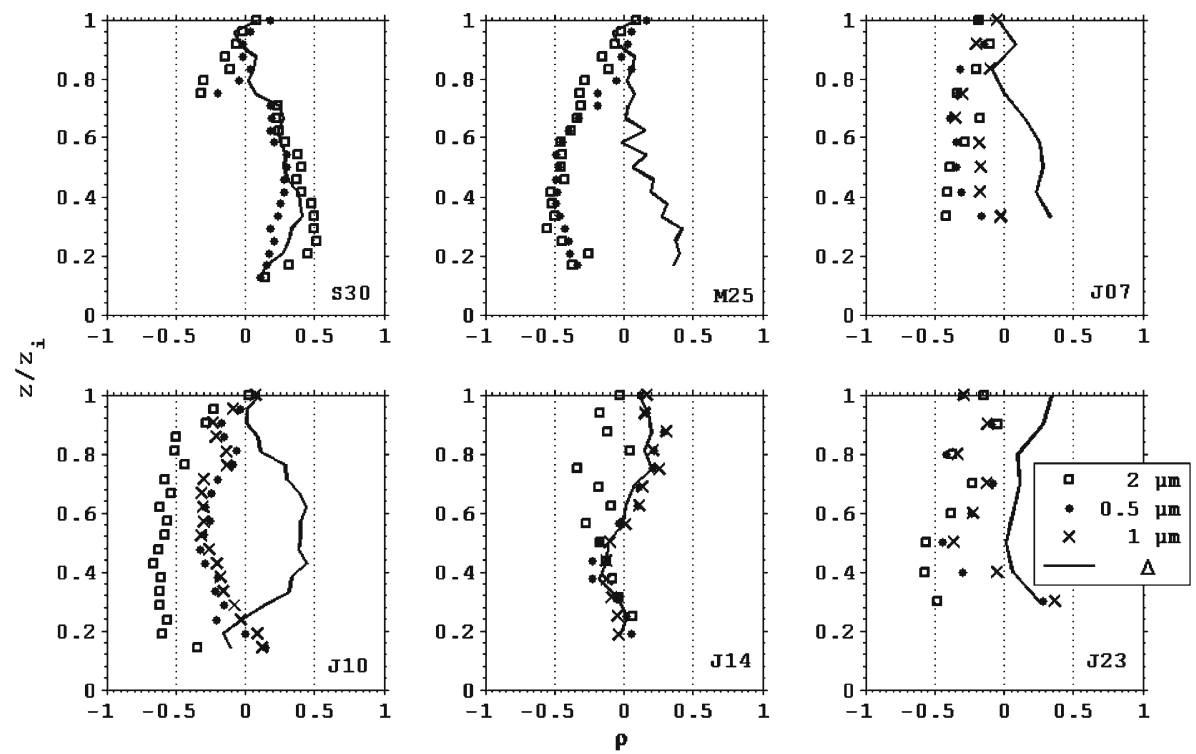

Fig. 10 Vertical profiles of correlations $\left(\rho\left(w, P z^{2}\right)\right.$ ) between the normalized lidar reflectivity $\left(P z^{2}-\left\langle P z^{2}\right\rangle\right) / \sigma\left(P z^{2}\right)$ (both from the 2,064 and $532 \mathrm{~nm}$ lidars), the depolarization ratio $\Delta$ and the vertical velocity $w$ by HDL at the same altitude. The altitude is normalized by the mean CBL height. Mean and standard deviations are calculated over a 1-h interval. The period of time used to calculate $\rho\left(w, P z^{2}\right)$ are indicated in Table 3 